\title{
A novel zero-watermarking scheme based on NSCT- SVD and blockchain for video copyright
}

\author{
Xiangqi Wu \\ Peng Ma \\ Hainan University \\ Zihan Jin \\ Hainan University \\ Yuxuan Wu \\ Hainan University \\ Wei Ou ( $\sim$ ouwei@hainanu.edu.cn ) \\ Hainan University \\ Wenbao Han \\ Hainan University
}

School of Cyber Security (School of Cryptology), Hainan University

\section{Research Article}

Keywords: Video copyright, Blockchain, Zero-watermarking, Privacy, Key-frames extraction

Posted Date: July 14th, 2021

DOI: https://doi.org/10.21203/rs.3.rs-288335/v2

License: (c) (i) This work is licensed under a Creative Commons Attribution 4.0 International License.

Read Full License

Version of Record: A version of this preprint was published at EURASIP Journal on Wireless Communications and Networking on March 21st, 2022. See the published version at https://doi.org/10.1186/s13638-022-02090-x. 
1 A novel zero-watermarking scheme based

2 on NSCT-SVD and blockchain for video

3 copyright

Xiangqi $\mathrm{Wu}^{1}$, Peng $\mathrm{Ma}^{1}$, Zihan $\mathrm{Jin}^{1}$, Yuxuan $\mathrm{Wu}^{1}$, Wei $\mathrm{Ou}^{{ }^{*}}$ and Wenbao $\mathrm{Han}^{1}$

*Correspondence: ouwei@hainanu.edu.cn

${ }^{1}$ School of Cyber Security (School of Cryptology), Hainan University, 58 Renmin Avenue, Meilan District, Haikou 570228, China

Full list of author information is available at the end of the article

\begin{abstract}
With the advent of information era, issues about video copyright continue to emerge. In the grey industrial chain of piracy, the film industry is a major disaster area so far. The current protection schemes for video copyright are inefficient and costly so that video industry still has to face the infringement. Research on a simple and efficient protection technology of video copyright is urgent. To address the inefficiency and irregularity of the current protection schemes for video copyright, this paper proposes a simple and efficient protection scheme for video copyright based on video zerowatermarking and blockchain. The results show that key-frames extraction and key-frames zerowatermarking based on NSCT-SVD in the proposed video zero-watermarking algorithm perform well and achieve low-cost protection for video copyright in practical environment. In addition, video zerowatermarking algorithm of proposed scheme works well with blockchain. Proposed scheme improves some extent on database centralization and evidential difficulties of traditional video zerowatermarking schemes, and it is more reliable, simpler and more effective than traditional video zerowatermarking schemes without blockchain.
\end{abstract}

Keywords: Video copyright, Blockchain, Zero-watermarking, Privacy, Key-frames extraction

\title{
1 Introduction
}

Since the turn of the millennium, the demand for video has risen quickly with the development of video products. However, some illegal video application damage the owners' copyrights. Against this background, a reliable protection scheme for video copyright has become extremely necessary and urgent in 21 st century.

The development of technology for copyright protection is closely related to the demand for video copyright protection. With video springing up as the main way of information dissemination all over the world, there is a greater need for protection scheme of video copyright. In particular, TikTok, YouTube and Hollywood, the three organizations that represent the three most dominant approaches in video industry today (such as short-form video, self-published video and digital cinema). They all have a quite 
strong need for reliable protection scheme of video copyright. Hollywood loses 64.6 billion dollars for pirated videos every year. TikTok and YouTube are even more so.

Unfortunately, in the world wide, the priority of current research is still more on traditional image copyright authentication and less on video copyright authentication. The video copyright authentication still needs to be developed. Considering of the application prospects of reliable protection scheme for video copyright, the urgency of copyright owner's demands and the importance of economy development, researches on video copyright protection are supported by a number of government agencies around the world.

With the continuous research on information hiding, digital watermarking technology of information hiding technology has been applied in the field of copyright protection. However, the traditional video blind watermarking schemes lack public credibility and are easy to be detected and attacked, and cannot be well qualified to verify video copyright. Traditional video zero-watermarking schemes are recognized by the trusted third-party. But in situation of the cyberspace security, the centralized management still has some problems. Therefore, zero-watermarking scheme with decentralized management is one of the best solutions. In conclusion, the main problems of existing watermarking schemes on video copyright protection are as follows.

- Poor robustness: There is a high probability that the watermark will be corrupted after multitransform in the current network architecture or compression. So that it becomes ineffective for video copyright.

- Weak imperceptibility: The addition of watermark to some videos has a visually perceptible impact on the original quality of the video, making it less commercially viable.

- Single function: Most current schemes have single function on copyright authentication. However, in an actual scenario of copyright protection, there will be some functions such as traceability of rights. The deficiency of existing schemes makes the actual process of rights maintenance quite complicated.

In proposed scheme, to solve these pressing issues about poor robustness and weak stealthy of existing protection schemes, we design a video key-frames extraction algorithm based on distance threshold clustering and a colour zero-watermarking algorithm based on combination of Non-Subsampled Contourlet Transform (NSCT) with Singular Value Decomposition (SVD). We innovatively combine video zero-watermarking algorithm with blockchain to address problem on data management and traceability of rights in current scheme for video copyright [1].

The remaining part of the paper is organized as follows. The related works are in Related works which introduces the development of digital watermarking applied to copyright protection, lists the examples about the new technology applied to copyright protection, and describes the watermarking algorithms proposed by related scholars detailed. The proposed scheme is elaborated in Scheme. Firstly, the overall architecture of system for video copyright protection is introduced, and how to combine zerowatermarking with blockchain to realize copyright protection function is explained. Secondly, we introduce the extraction algorithm of the key-frames from video. Thirdly, we introduce a zerowatermarking algorithm on key-frames based on NSCT and SVD. Results shows the experimental results 
of proposed scheme, and the reliability is verified. The security and privacy of proposed scheme are discussed in Discussion. Lastly, works of this paper are concluded in Conclusions.

\section{Related works}

Among the techniques which are used to protect digital intellectual property, digital watermarking of information hiding technology is the most popular. The creation of digital watermarking could be dated back to 1954, when Emil Hembrooke of Muzak applied for a patent. The patent is applying a narrowband trap filter with a central frequency of $1 \mathrm{kHz}$ to insert a verification code. This system was used by Muzak until 1984. Research on digital watermarking began in the early 1990s. The concept of digital watermarking was first introduced at the International Conference on Image Processing in 1994 [2], and then the First International Symposium on Information Hiding was held in the UK in 1996 [3]. At the Third International Symposium on Information Hiding in 1999, digital watermarking dominated the conference, with 18 of the 33 papers were devoted to the study of digital watermarking. Currently, digital watermarking has evolved towards copyright protection for any types of digital files.

In the previous works, scholars from all over the world have made a great progress in the study of digital watermarking on images. KOBAYASHI Atsushi, HASEGAWA Akria and et al. propose that digital watermarking algorithms can be embedded in edge devices as a complement to other permission management and they investigate the application of digital watermarking to both still images and moving images [4]. He Fang studies on wavelet-based high-frequency and low-frequency methods for inserting watermark and tests their anti-compressibility for watermark extraction. The experiments show that the insertion of watermark in the high frequency domain keeps the original quality better, and the insertion of watermark in the low frequency domain makes the robustness better [5]. Zai-rong WANG, Babak SHIRI's innovatively use fractional logistic mapping for generating chaotic sequences, then encrypt and insert the watermark into the original image. Because the fractional order of the encrypted image is within $(0,1]$, the key space is increased [6]. Their research provides a robust and safe watermarking method in the protection of the copyright of hardware, image and other electronic documents [6]. Yuan-Min Li and Deyun Wei propose a watermarking algorithm with double encryption based on cosine transform and fractional Fourier transform in the invariant wavelet domain. Their work innovatively utilises image magnification technique to pre-process the host image to enhance embedding capacity of the watermarking algorithm. They use the redistribution-invariant wavelet transform and the discrete cosine transform to obtain the hybrid domain, and they also propose a multi-parameter particle swarm optimisation algorithm, which is used to obtain the optimal embedding factor matrix [7].

Zero-watermarking [8] has become a focus of research in recent years since it was proposed by Wen Quan, as it did not make any modification to the original digital file. Zhiqiu Xia and Xingyuan Wang et al. propose multiple zero-watermarking scheme based on local quaternion polar harmonic Fourier moments (QPHFMs) for colour medical images, and the construction of multiple zero-watermarking effectively improves reliability and resists complex out-of-step attacks, such as clipping [9]. Hongfei Xue and Wanda Chi propose a more robust zero-watermarking algorithm based on NSST, DCT and Hessenberg decomposition. They innovatively generate a zero-watermark by performing XOR between the QR code and the characteristic matrix of a colour image [10]. In recent years, the combination of deep learning and watermarking algorithm has been also a key research area. Ali Amiri and Satter Mirzakuchaki propose an intelligent watermarking model based on NSCT-SVD. They innovatively use 
PSO-GA-AI algorithm, and the obtained PSO and PSO-GA algorithms with larger SF have higher stability, then low frequency coefficient is fed back to the SVD through NSCT and SWT so that the values of model visual transparency and PSNR will increase [11].

Video is a very important part of the digital media. Currently, there are three types of schemes about video watermarking. The first way is the watermarking scheme based on original video, which is about embedding the information of watermark into the original video. It should be noted that the first way is our choice in proposed scheme. The second way is based on the video encoding process. The compression standards of mainstream video include the MPEG series of ISO/IEC and the H.264 series of ITU-T [12,13], and the key technology to achieve a high compression ratio is predictive coding and transform coding. The disadvantage of the second way is the presence of cumulative errors which can have an adverse effect on video quality [14]. The third way is based on the compressed domain. Because the video is currently transmitted and stored after compression, the third way is based on the compressed domain. The advantage of this way is that the embedded watermarking doesn't need to be re-encoded and decoded, so it can reduce computational complexity through this way. The disadvantage is that the low redundancy of the compressed data limits the amount of data that can be watermarked, and the embedding strength is limited by the encoding of the video data. Y Sun and J Wang remedy the shortcomings of the existing watermarking algorithms on H.264/SVC framework. Their research uses DCT (the discrete cosine transforms) and an invisible watermarking framework with scalable watermarking algorithms to accommodate the multi-resolution of H.264 / SVC, and they design control mechanisms of key to select sub-blocks adaptively from macroblocks [15]. Their proposed method achieves no less than $41 \mathrm{~dB}$ peak signal-to-noise ratio (PSNR) and a normalised correlation (NC) of $>0.85$ [15]. Yicheng Qiu and Feng Xue et al. propose a novel method of video watermarking for HEVC (High Efficiency Video Coding) that the blocks for watermarking embedding are selected based on the temporal and spatial characteristics of the compressed video, which minimizes the synchronization error and can well prevent collusion attacks [16]. Besides, the public key is extracted from features of compressed domain to select the blocks for embedding, and the watermark is embedded on the blocks which reduces the computational overhead [16].

Except using digital watermarking for copyright protection, PetaPixel develops Blockkai which uses the Bitcoin blockchain to protect copyright, and Baidu's "Totem" uses blockchain technology to solve the pain point of image copyright [17]. Tencent Cloud uses blockchain technology to launch the "Zhixin Chain" to solve the problem of original content protection. Byte Dance's Tuchong has a deep partnership with Ant Blockchain of Ant Financial. Currently, Tuchong becomes the leading platform based on blockchain for the identification and ownership of photographers and the copyright of original works can be checked on the chain [18].

\section{Methods}

\subsection{Structure}

In this part, we will introduce how does the video zero-watermarking algorithm work with blockchain for protecting copyright. First of all, the main processes of registration about video copyright are as follows.

- Step 1. User A uploads a video and a colour image which is used for identity authentication, then 
authenticates identity through the terminal.

- $\quad$ Step 2. Pre-process the video data and extract the key-frames by distance threshold clustering.

- $\quad$ Step 3. Construct zero-watermarks on extracted key-frames.

- Step 4. Collect zero-watermarks, information of key-frames and Key $K_{2}$, then package these data.

- Step 5. After the terminal constructs and signs the data package into a proposal, the proposal is sent to the access node in the blockchain network, and the access node broadcasts the proposal after verification.

- Step 6. After receiving the block, the node verifies the proposal and composes the proposal into a block to be verified, and sends it to each node for processing. When more than two thirds of the nodes reach a consensus, the nodes start to generate blocks, then send them to blockchain network.

- Step 7. Blockchain network checks information of block (for example, block height) and each node will store a complete block and table data.

- Step 8 . The terminal returns the Key $K_{1}$ to User A by terminal.

Secondly, this proposed scheme uses the blockchain to realize the transactions of copyright. Compared with complicated operations in other solutions, the blockchain is more practical, and it is born to solve the trading problems. The main processes of copyright transaction are as follows.

- Step 1. User B sends a proposal, which is constructed and signed by terminal and sent to the access node.

- Step 2. The consensus node receives the proposal and takes out a number of proposals from pool in the order of the queue. After that, it will assemble them as consensus blocks and then send them to each node for processing.

- Step 3. After the node receives the block, the proposal is taken out of the block and executed in order, and the results of execution are packaged in the transaction receipt and returned.

- Step 4. The nodes exchange their execution results. When more than $2 / 3$ of the nodes have the same execution result, the node will start to generate blocks and permanently storage the transaction and execution results to hard disk.

Thirdly, the main processes for certification on video copyright are as follows.

- Step 1. In order to authenticate the video, User A needs to provide Key $K_{1}$ and the block hash to match other information on the blockchain to get the information of key-frames, zero-watermarks and Key $K_{2}$ on the chain.

- Step 2. Use the watermark extraction algorithm to return a colour watermark which can authenticate the identity and complete the authentication.

- Step 3. User B can simply check ledger on the blockchain network when he completes the transaction to prove his copyright of the video.

The above processes are described in Fig. 1. 


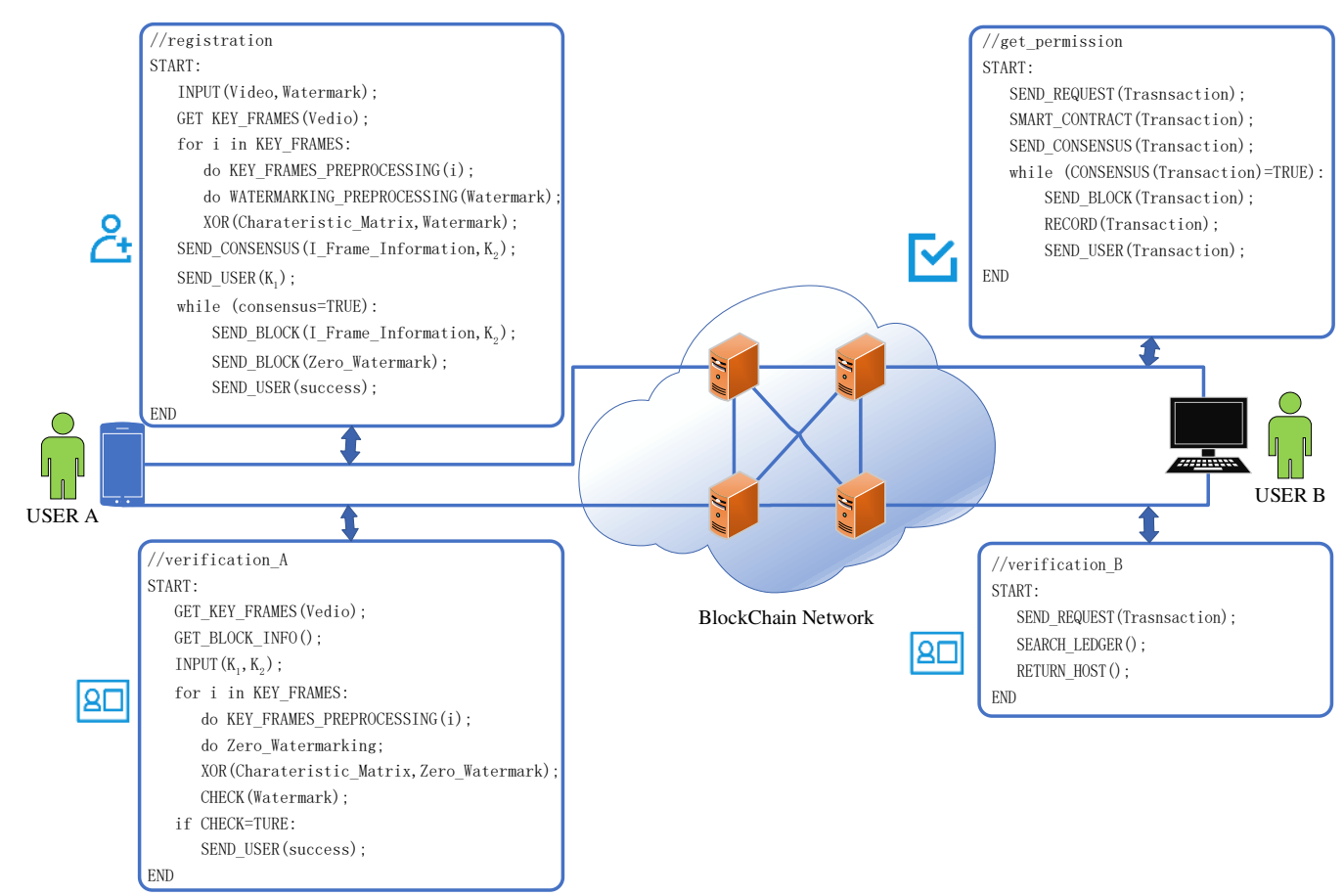

Fig. 1 Architecture of the protection system

\subsection{Key-frames extraction}

2 The main processes of key-frames extraction algorithm are as follows.

- $\quad$ Step 1. Input video samples and process the video library.

- Step 2. Convert unstructured dynamic video into a static sequence of video frames.

- Step 3. Process the samples initially and extract the feature vectors.

- Step 4. Calculate the inter-frame similarity and distance threshold according to the extracted feature vectors to complete the clustering.

- Step 5. Judge key-frames according to the clustering results.

- $\quad$ Step 6. Output the extracted key-frames.

\subsubsection{Feature extraction}

The selection of feature is very important to the expression of key-frames and the completion of effective clustering. Colour and texture features are common image features. At present, most of the key-frames extraction algorithms are based on clustering use colour histogram, but the feature of colour histogram cannot describe colour space distribution between adjacent pixels. As a local feature of an image, texture feature does not depend on colour and brightness. It not only reflects the grey level statistics of the image, but also reflects the spatial distribution information and structure information of the image. Its grey level invariability and rotation invariant can cleverly avoid experimental errors caused by significant changes in light. Therefore, in this paper, the completed LBP (CLBP) [19] descriptor of the improved local binary patterns (LBP) [19] is chosen to handle the texture features. CLBP texture descriptor [20] is an extended 
texture descriptor based on LBP descriptor [21], which can effectively describe the missing information of LBP type in order to obtain better performance of texture classification.

Usually, given a central pixel and other $\mathrm{P}$ neighbourhood values, the local difference vector is expressed as $\left[d_{0} \ldots \ldots d_{P-1}\right], d_{p}=g_{p}-g_{c} . s_{p}$ and $m_{p}$ are the sign (positive or negative) and magnitude values of $d_{p}$ using the local difference sign-separation-magnitude transform (LDSMT), respectively. The above content is shown as follows:

$$
\mathrm{d}_{P}=s_{p} m_{p} \text { and }\left\{\begin{array}{c}
s_{p}=\operatorname{sign}\left(\mathrm{d}_{P}\right) \\
m_{p}=\left|\mathrm{d}_{p}\right|
\end{array}\right.
$$

and

$$
s_{p}=\left\{\begin{array}{c}
1, d_{p} \geq 0 \\
-1, d_{p}<0
\end{array}\right.
$$

The above formulas cannot be used directly as a descriptor because it is sensitive to light, rotation, noise, etc. The difference between the neighbouring pixels and the central pixel is divided into a sign component and a gradient component and is obtained by multiplying the two together, denoted as CLBP_S and CLBP_M.

For example, Fig. 2a is a sampling block which is size of $3 \times 3$, which can be divided into Fig. $3 a \times$ Fig.3b, and its difference can be represented by Fig. 2b. Fig. 3a is sign components, and Fig. 3b is gradient components. Actually, CLBP_S is the traditional LBP, and the two coding methods are the same. -1 in Fig. $3 \mathrm{a}$ is equal to 0 in the traditional LBP. What's more, the CLBP_S is more capable to describe texture than the CLBP_M. Formula of CLBP_S is shown as follows:

$$
C L B P \_S_{P, R}=\sum_{P=0}^{P-1} s\left(s_{p}\right) 2^{p}
$$

and

$$
s(x)=\left\{\begin{array}{l}
1, x \geq 0 \\
0, x<0
\end{array}\right.
$$
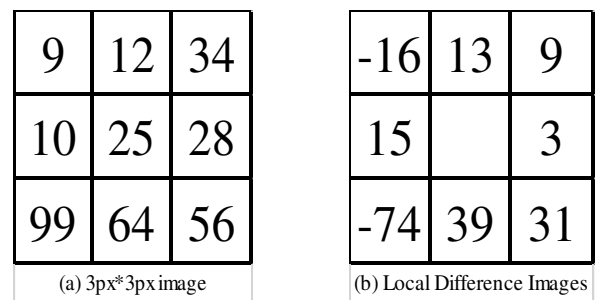

Fig. 2 Description of CLBP algorithm I
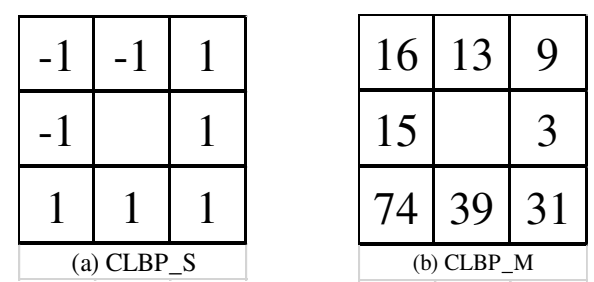

Fig. 3 Description of CLBP algorithm II 


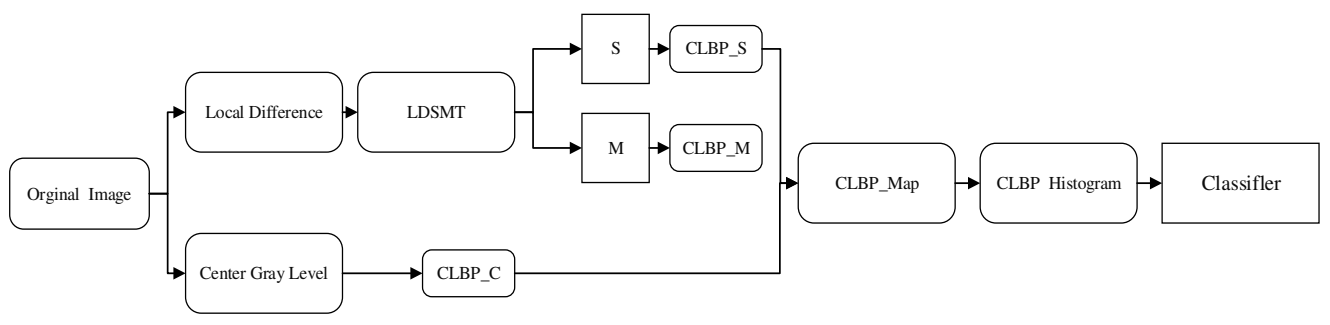

\section{Fig. 4 CLBP algorithm}

It can be seen from Fig. 3b, CLBP_M is a continuous value. In order to harmonise the encoding with CLBP_S, it needs to be converted to binary encoding as well. Inspired by CLBP_S, this paper follows the following encoding for them, $\mathrm{c}$ is an adaptive threshold, and the value of $\mathrm{c}$ is taken as the mean value of $m_{p}$ in the whole image. $m_{p}$ is the absolute value accumulation of gradient differences between neighbourhood pixels and centre pixels (or the average of them, the final results of comparison are equal). Formula of CLBP_M is shown as follows:

$$
C L B P_{-} M_{P, R}=\sum_{p=0}^{p-1} t\left(m_{p}, c\right) 2^{p}
$$

and

$$
t(x, c)=\left\{\begin{array}{l}
1, x \geq c \\
0, x<c
\end{array}\right.
$$

The central pixel represents the local grey level and also contains the local grey level discriminant information. In order to effectively combine it with CLBP _ M and CLBP_S, we carry out the following encoding:

$$
C L B P_{-} C_{P, R}=t\left(g_{c}, c_{I}\right)
$$

and

$$
t(x, c)=\left\{\begin{array}{l}
1, x \geq c \\
0, x<c
\end{array}\right.
$$

In Eq. 7, $g_{c}$ represents the centre pixel grey value, $c_{l}$ represents the average grey value of the whole image. Binary encoding is performed by comparing the centre pixel and the average pixel value of the entire image.

The process of CLBP algorithm is shown in Fig. 4. After the above calculation, the three descriptors CLBP _ S, CLBP _ M, CLBP _ C are all generated, which can be fused in the form of series, parallel or series-parallel histogram, and can achieve more effective rotation invariant classification ability than the traditional LBP.

\subsubsection{Inter-frame similarity}

Distance measurement is used to measure the distance between individuals in space. The farther the distance is, the greater the difference between individuals is, and the lower the similarity is. Chi-square distance is the most common measure to measure the difference between two individuals, and its calculation is simple. Therefore, in this paper we use chi-square distance to represent the similarity between frames. The smaller the chi-square distance is, the higher the similarity between frames is. The chi-square distance between any two frames is defined as follows: 
In Eq. 9, $F_{a}[i]$ and $F_{b}[i]$ represent the values of image a and image $\mathrm{b}$ on the binary bit $\mathrm{i}$. Respectively, $n$ is the total number of video frame histogram bins, and $\operatorname{dist}\left(F_{a}, F_{b}\right)$ represents the inter-frame similarity.

\subsubsection{Distance threshold}

The distance threshold directly affects the number of clusters, thereby affecting the extraction efficiency of key-frames. If the threshold is too small, it is easy to extract too many key-frames, resulting in information redundancy. If the threshold is too large, the extracted key-frames cannot represent the main content of the lens. According to the idea of data density sampling [23], the distance threshold is defined as follows:

$$
d=\frac{2 c}{N(N-1)} \sum_{i=1}^{N-1} \sum_{j=i+1}^{N} \operatorname{dist}\left(F_{i}, F_{j}\right)
$$

In Eq. 10, $\mathrm{N}$ is the total number of video frames, and $\frac{N(N-1)}{2}$ is the number of chi-square distances between two $\mathrm{N}$ frames. $\mathrm{c}$ is a constant. A large number of experiments show that the value of 0.25 in this algorithm is better. $\mathrm{d}$ is the distance threshold of clustering algorithm obtained by density sampling. By this method, different thresholds can be selected for different videos, which reflects the adaptability of the threshold. The threshold selected by this method will be small but not too small, and appropriate small threshold can obtain more initial clustering, which is conducive to cluster centre merging and secondary clustering.

\subsubsection{Description of clustering}

All the steps of algorithm description are shown as follows.
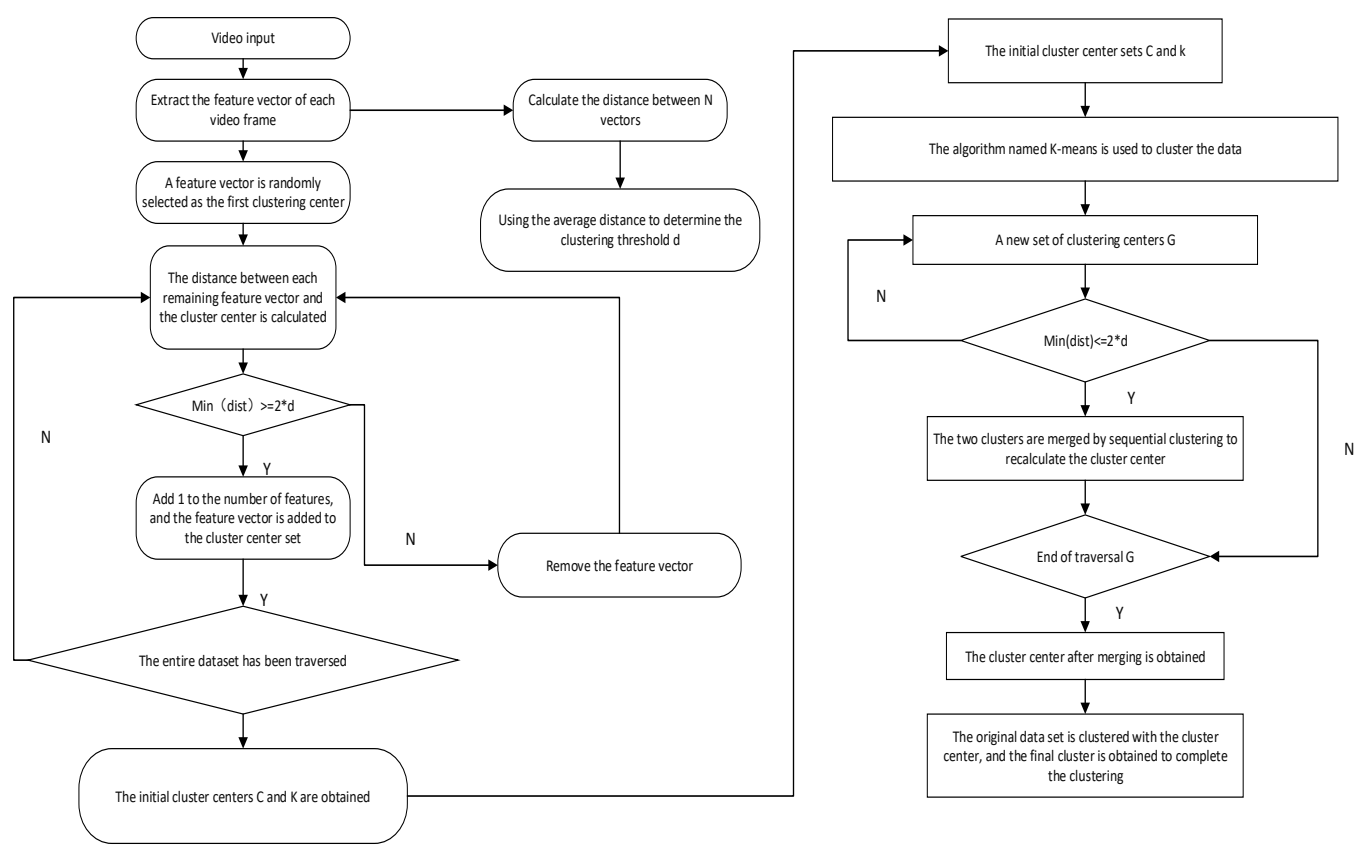

Fig. 5 Process of clustering algorithm 
- $\quad$ Step 1. Sample the data set to determine the distance threshold d.

- $\quad$ Step 2. Initial clustering of data sets is based on threshold and similarity, and then determine the initial number of clusters $\mathrm{k}$ and cluster centre set $\mathrm{C}$.

- $\quad$ Step 3. Use K-means algorithm to optimize the initial clustering centre, then we get new sets of clustering centre $\mathrm{G}$.

- $\quad$ Step 4. Use the sequential clustering idea [24] to merge the closer classes in G, then determine the final $\mathrm{k}$ and cluster centre set and cluster, and then complete the clustering centre selfdetermination.

Process of clustering algorithm is shown in Fig. 5 (step 1 and step 2 are on the left. Step 3 and step 4 are on the right). After clustering, we select all the nearest frames from the clustering centre to be the key-frames of the video.

\subsection{Zero-watermarking for key-frames}

After the digital image has been SVD [25-27], the corresponding orthogonal matrix represents the geometric structure of the image while the corresponding singular matrix represents the image luminance information. The real number field we define is R. Assuming the digital image matrix is $\mathrm{A}$ and $\mathrm{A} \in R_{N \times N}$, then Orthogonal matrix $\mathrm{U} \in R_{N \times N}$, orthogonal matrix $\mathrm{V} \in R_{N \times N}$ and diagonal matrix $\mathrm{S} \in R_{N \times N}$. Therefore, A can be expressed as:

$$
A=S \times U \times V^{T}
$$

$\mathrm{U}$ is defined as the left orthogonal matrix and $\mathrm{V}$ is defined as the right orthogonal matrix. They satisfy the conditions respectively, which are $U \times U^{T}=1, V=V^{-1}$. In that $\mathrm{S}$ :

$$
\mathrm{S}=\left(\begin{array}{ccc}
\lambda_{1} & 0 & 0 \\
0 & \ddots & 0 \\
0 & 0 & \lambda_{\mathrm{r}}
\end{array}\right)
$$

A special singular value in the $\mathrm{S}$ matrix is usually much larger than other singular values, which can represent the overall energy of A [28].

The Arnold Transform [29-31] can change the position of pixels and eliminate correlations between pixels or content in a host image, thus improving image security. The special Arnold Transform is the Simplest kind of Arnold Transform. The formula for operation of matrix is:

$$
\left(\begin{array}{l}
x^{\prime} \\
y^{\prime}
\end{array}\right)=\left[\begin{array}{ll}
1 & 1 \\
1 & 2
\end{array}\right]\left(\begin{array}{l}
x \\
y
\end{array}\right) \bmod (N)
$$

The special Arnold Inverse Transform makes it easy to recover the transformed image. The formula for operation of matrix is:

$$
\left(\begin{array}{l}
x \\
y
\end{array}\right)=\left[\begin{array}{cc}
2 & -1 \\
-1 & 1
\end{array}\right]\left(\begin{array}{l}
x^{\prime} \\
y^{\prime}
\end{array}\right) \bmod (N)
$$

$\left(x^{\prime}, y^{\prime}\right)$ denotes the position of $(x, y)$ at the pixel point after the transformation and $\mathrm{N}$ denotes the size of the image involved in the transformation.

\subsubsection{Watermark generation}

After using the key-frames extraction algorithm based on distance threshold clustering, a series of keyframes are selected from the video which represent the feature information of the whole video. The zero- 
watermarking algorithm is extracting the feature information of these key-frames and embedding the feature information into the watermark later. The detailed steps are as follows.

- Step 1. Output the key-frames sequence obtained by running the key-frames extraction algorithm of the previous part, denoted as Frame I. The size of the frame is $M \times N \times 3$.

- Step 2. Perform RGB decomposition on the key-frames and get three grey-scale images, denoted as $I_{R}, I_{G}, I_{B}$. Then make $I_{R}, I_{G}, I_{B}$, the three grey-scale images into $A \times B$ non-overlapping chunks (Operations on the separated three frames are the same and we will only introduce one in the following). Suppose the values of M and N are both 512, and the values of A and B are both 128. The chunking method is as follows.

$$
B_{i, j}=\frac{I_{k}^{M \times N}}{A \times B}, k \in\{R, G, B\}, i, j \in\{1,2,3,4\}
$$

- Step 3. Do Arnold Transform on each sub-block, and the disordering key is Key $K_{1}$. Assume that the chunked sub-blocks are transformed according to Eq. 13. The Arnold Transform will disrupt correlation between pixels of the grey-scale.

- $\quad$ Step 4. Do NSCT for each sub-block $B_{i, j}$ after scrambling. The NSCT yields low-frequency information $c A_{i, j}$ for each sub-block containing point singularities and curve singularities. Following the assumptions in step 2, The size of $c A_{i, j}$ is $32 \times 32$.

- Step 5. Subdivide the low frequency component $c A_{i, j}$ again and subdivide $c A_{i, j}$ into sub-blocks of $4 \times 4$ size. The chunking method is as follows.

$$
c B_{m, n}=\frac{c A_{i, j}}{8 \times 8}, m, n \in\{1, \ldots, 8\}, i, j \in\{1, \ldots, 4\}
$$

- Step 6. Do SVD [32] on each sub-block according to Eqs. 8 and 9. The method is as follows.

$$
\left[U_{i, j}, S_{i, j}, V_{i, j}\right]=\operatorname{SVD}\left(c A_{i, j}\right)
$$

Owing to the highest bit of each singular value matrix of each sub-block does not change under a certain range of attacks, the maximum singular value of each singular value matrix is chosen to construct the characteristic matrix X. After SVD, characteristic matrixes $\left\{X_{R}, X_{G}, X_{B}\right\}$ can be get. Characteristic matrix $\mathrm{X}$ is as follow.

$$
x=\left[\begin{array}{ccc}
f_{1,1} & \cdots & f_{1,32} \\
\vdots & \ddots & \vdots \\
f_{32,1} & \cdots & f_{32,32}
\end{array}\right]
$$

- Step 7. Process a meaningful watermark image, and its size is $m \times n \times 3$. After the RGB decomposition, get the three grey-scale images, denoted as $I_{R}{ }^{\prime}, I_{G}{ }^{\prime}, I_{B}{ }^{\prime}$, then do Arnold Transform on $I_{R}{ }^{\prime}, I_{G}{ }^{\prime}, I_{B}{ }^{\prime}$ and it's the distorting key is Key $K_{2}$. Assume the disordered frames are $W_{R}{ }^{\prime}, W_{G}{ }^{\prime}, W_{B}{ }^{\prime}$.

- Step 8. Do NSCT on $W_{R}{ }^{\prime}, W_{G}{ }^{\prime}, W_{B}{ }^{\prime}$, then get high frequency parts which are denoted as $H_{R}{ }^{\prime}$, $H_{G}{ }^{\prime}, H_{B}{ }^{\prime}$ and the low frequency parts which are denoted as $L_{R}{ }^{\prime}, L_{G}{ }^{\prime}, L_{B}{ }^{\prime}$. Run XOR $\left\{H_{R}{ }^{\prime}\right.$, $\left.H_{G}{ }^{\prime}, H_{B}{ }^{\prime}\right\}$ and $\left\{L_{R}{ }^{\prime}, L_{G}{ }^{\prime}, L_{B}{ }^{\prime}\right\}$ with each of the three channels of the characteristic matrixes $\left\{X_{R}, X_{G}, X_{B}\right\}$ obtained in step 6 . The method is as follows.

$$
\begin{aligned}
Z_{K H} & =\operatorname{XOR}\left(X_{k}, H_{K}{ }^{\prime}\right), K \in\{R, G, B\} \\
Z_{K L} & =X O R\left(X_{k}, L_{K}{ }^{\prime}\right), K \in\{R, G, B\}
\end{aligned}
$$




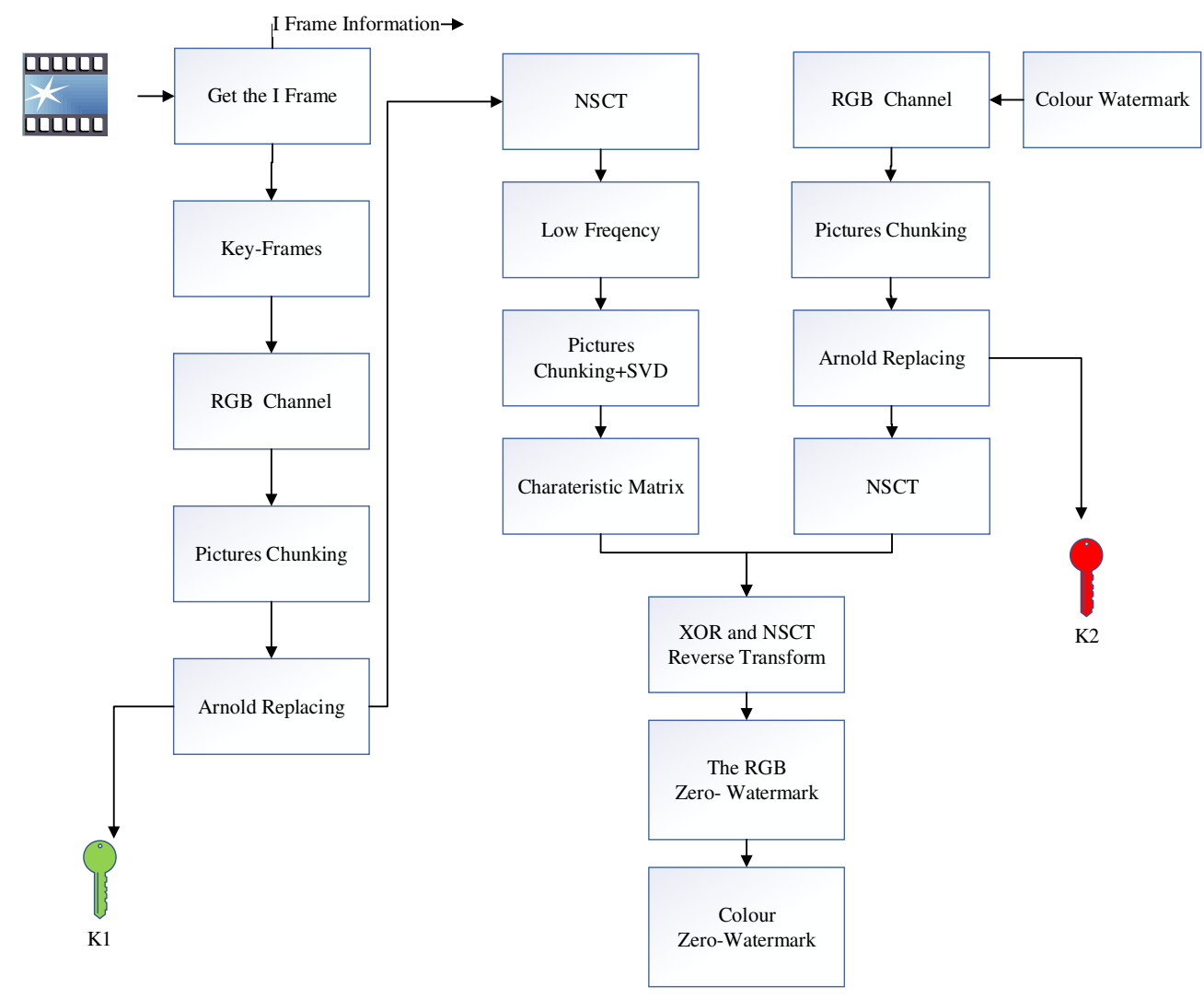

Fig. 6 Process of watermark generation

- Step 9. Do the inverse NSCT on $Z_{K H}$ and $Z_{K L}$, then composite RGB channels to produce a colour zero-watermark. The method is as follows.

$$
\begin{gathered}
Z_{K H}+Z_{K L}=Z_{K}, K \in\{R, G, B\} \\
Z_{R, G, B}=Z_{R}+Z_{G}+Z_{B}
\end{gathered}
$$

All the above steps are shown in Fig. 6.

\subsubsection{Watermark extraction}

The extraction method is organized as follows.

- Step 1. Based on the information of the Frame I, obtain the sequence of key-frames.

- $\quad$ Step 2. Follow step 2 in Watermark generation for RGB decomposition and chunking.

- $\quad$ Step 3. Use Key $K_{1}$ to do Arnold Transform like step 3 in above watermark generation for each of the chunked sub-blocks.

- Step 4. Follow step 4, step 5 and step 6 in above Watermark generation. After those Steps, characteristic matrixes which are denoted as $X_{R}{ }^{\prime}, X_{G}{ }^{\prime}, X_{B}{ }^{\prime}$ are generated.

- Step 5. Process colour zero-watermark, and it is size of $m \times n \times 3$. After the RGB decomposition, the separated frames which are denoted as $I_{R} ", I_{G} ", I_{B} "$. Do NSCT on $I_{R} ", I_{G} ", I_{B} "$, then get the high frequency parts which denoted as $H_{R}{ }^{\prime}, H_{G}{ }^{\prime \prime}, H_{B}$ " and the low frequency parts which

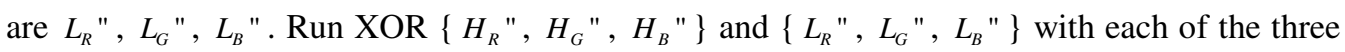
channels of the characteristic matrixes $\left\{I_{R} ", I_{G} ", I_{B} "\right\}$. The method is as follows: 

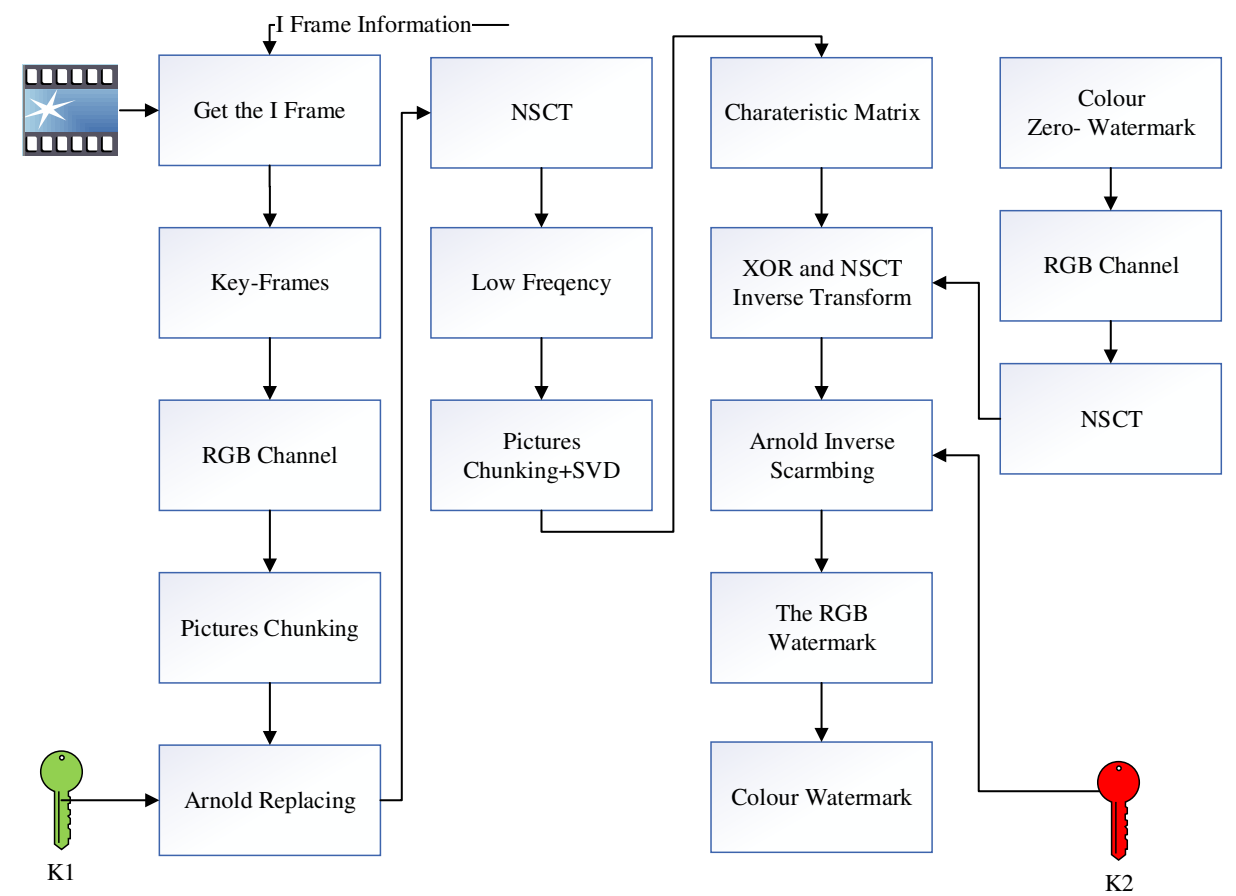

Fig. 7 Process of watermark extraction

$$
\begin{aligned}
& V_{K H}{ }^{\prime}=\operatorname{XOR}\left(X_{k}{ }^{\prime}, H_{K}{ }^{\prime \prime}\right), K \in\{R, G, B\} \\
& V_{K L}{ }^{\prime}=\operatorname{XOR}\left(X_{k}{ }^{\prime}, L_{K}{ }^{\prime \prime}\right), K \in\{R, G, B\}
\end{aligned}
$$

- Step 6. Do the inverse transform of NSCT on $V_{K H}{ }^{\prime}$ and $V_{K L}{ }^{\prime}$.

$$
V_{K H}{ }^{\prime}+V_{K L}{ }^{\prime}=V_{K}{ }^{\prime}, K \in\{R, G, B\}
$$

- Step 7. Use the Key $K_{2}$ which is kept by the owner of video copyright to do the Arnold Inverse Transform, then we get $Z_{R, G, B}$ ' from $V_{R, G, B}$ ' by Arnold Inverse Transform. The method is similar to Eq. 14. Finally, composite three grey-scale images to produce a colour watermark. The method is as follows.

$$
Z_{R, G, B}{ }^{\prime}=Z_{R}{ }^{\prime}+Z_{G}{ }^{\prime}+Z_{B}{ }^{\prime}
$$

All the above Steps are shown in Fig. 7.

\section{Results}

In this section, we test on the three parts of proposed scheme, which are blockchain system, key-frames extraction algorithm based on distance threshold clustering and zero-watermarking algorithm for keyframes based on NSCT-SVD.

The purpose of testing blockchain system is to actually explore whether the poor blockchain can be applied to the protection for video copyright. Furthermore, it is worth emphasizing that video zerowatermarking consists of key-frames extraction algorithm and zero-watermarking algorithm. Therefore, the purpose of testing efficiency key-frames extraction algorithm and zero-watermarking algorithm are 
1 to verify the process of extracting the features on key-frames in the video and obtained zero-watermark

2 which was recorded on blockchain is robust and secure.

\section{$3 \quad 4.1$ Experiment deployment}

4 The hardware configuration of the whole experiments is shown in Table 1, and the software configuration

5 of the whole experiments is shown in Table 2.

Table 1 Hardware configuration

\begin{tabular}{ll}
\hline Environmental parameters & Config \\
\hline CPU & Intel i7, 2.4GHz \\
GPU & GTX1080 Ti 11GB \\
RAM & $16 G B$ \\
Mainboard & Z270-AR \\
Hard disk & 500GB SSD + 2T HDD \\
\hline
\end{tabular}

Table 2 Software configuration

\begin{tabular}{ll}
\hline Environmental parameters & Config \\
\hline OS & Windows 10 \\
Host Environment & Ubuntu 18.04, 64bit \\
Database & MySQL 8.0.18 \\
Blockchain platforms & Fisco Bcos \\
Python & 3.7 .4 \\
MATLAB & R2019b \\
\hline
\end{tabular}

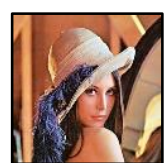

(a) lena

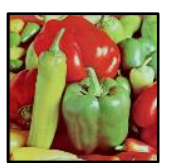

(b) vegetable

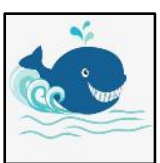

(c) animal

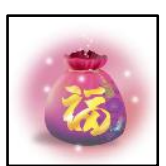

(d) bag

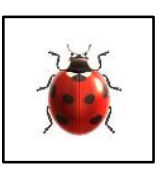

(e) ant

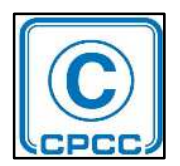

(f) CPCC

Fig. 8 Images and copyright watermark

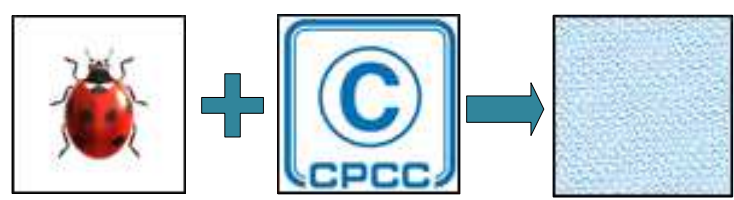

(a)

(b)

(c)

Fig. 9 Zero-watermark after extraction 
Table 3 Video key-frames extraction experimental results

\begin{tabular}{lllll}
\hline & Landscapes & Advertisement & Animation clips & News \\
\hline $\begin{array}{l}\text { Total } \\
\text { frames }\end{array}$ & 115 & 1254 & 15324 & 26987 \\
CF & 14 & 135 & 735 & 1452 \\
Df & 1 & 7 & 52 & 182 \\
MF & 0 & 2 & 23 & 85 \\
R /\% & 100 & 98.54 & 96.97 & 94.47 \\
P /\% & 93.33 & 95.07 & 93.39 & 88.86 \\
\hline
\end{tabular}

In process of simulating the key-frames extraction algorithm, we choose videos with unequal length to test the actual effect of the key-frames extraction algorithm, which are Landscapes, Advertisement, Animation clips and News respectively.

In order to prove the false alarm, robustness and security of proposed zero-watermarking algorithm for key-frames based on NSCT-SVD, five images are selected to replace key-frames in experiments, which are Figs. 8(a)-8(f). Fig. 8(f) is defined as copyright watermark in experiment. All the figures are shown in Fig. 8. In Fig. 9, it is an example of generated Fig. 9(c) which is a zero-watermark constructed by Figs. 9(a) and 9(b).

\subsection{Key-frames extraction}

The zero-watermarking algorithm we proposed is to construct a zero-watermark based on the extracted key-frames. So, this part tests the effectiveness of video key-frames extraction algorithm which is based on K-means clustering algorithm.

This part of experiment has the following definitions: Recall ratio is denoted as $\mathrm{R}$ and Precision ratio is denoted as P. Correctly detection is CF. Missed detection is MF. False detection id DF. R and P are calculated as follows.

$$
\begin{aligned}
& R=\frac{C F}{C F+M F} \\
& R=\frac{C F}{C F+M F} \\
& P=\frac{C F}{C F+D F}
\end{aligned}
$$

The experimental results are shown in Table 3.

\subsection{Zero-watermarking}

This part reproduces the zero-watermarking algorithm based on NSCT-SVD with the images described in above, and tests the performance of the algorithm in different scenarios from false alarm, robustness, and security.

\subsubsection{False alarm}

The experiment content is as follows. In this experiment, we use the five images which are Figs. 8(a)8(e) to generate the corresponding zero-watermark with the copyright watermark, and then calculate 
Table 4 NC of zero-watermark between different images

\begin{tabular}{cccccc}
\hline Host Image & Lena & Vegetable & Bag & Ant & Animal \\
\hline Lena & 1.0000 & 0.5769 & 0.5125 & 0.4643 & 0.5571 \\
Vegetable & 0.5769 & 1.0000 & 0.4517 & 0.4943 & 0.3753 \\
Bag & 0.5125 & 0.4517 & 1.0000 & 0.5360 & 0.4777 \\
Ant & 0.4643 & 0.4943 & 0.5360 & 1.0000 & 0.5570 \\
Animal & 0.5571 & 0.3753 & 0.4777 & 0.5570 & 1.0000 \\
\hline
\end{tabular}

Table 5 Comparation between DWT-SVD and NSCT-SVD

\begin{tabular}{ccc}
\hline Attack Types & DWT-SVD & NSCT-SVD \\
\hline Sault and pepper noise (0.03) & 0.9669 & 0.9870 \\
Gaussian noise (0.03) & 0.9643 & 0.9785 \\
Multiplicative noise (0.03) & 0.9712 & 0.9915 \\
Median filtering $(5 * 5)$ & 0.9709 & 0.9966 \\
Mean filtering $(5 * 5)$ & 0.9752 & 0.9944 \\
Wiener filtering $(5 * 5)$ & 0.9781 & 0.9987
\end{tabular}

normalized correlation (NC) between the five zero-watermarks. NC value reflects false alarm rate. The experimental results are shown in Table 4.

\subsubsection{Robustness}

In this part, we discuss robustness on the proposed zero-watermarking algorithm from three parts which are noise attack, filtering attack, geometric attack.

Before each attacking test on the proposed zero-watermarking algorithm, we select Fig. 8(a) to compare traditional DWT-SVD and NSCT-SVD under noise attacks and filtering attacks. We also calculate the normalized correlation between Fig. 8(a) and extracted copyright watermark after attacks. The results are shown in Table 5 .

Afterwards, we test the robustness of the zero-watermarking algorithm for key-frames under noise attacks, filtering attacks and cropping attacks. The results are shown in Fig.10.

As seen from Fig. 10(a), 10(b) and 10(c), the watermarks keep visual stability well after extraction under three noise attacks. In addition, it can be seen from Fig. 10(d) that the watermark extracted after sharpening attack is still very clear. Common geometric attacks include cropping attacks, rotation attacks and row-column offsets. We have cropped one-16th and one-32th respectively in the upper left corner of the picture to test the robustness under geometric attacks. The results are shown in Figs. 10(e) and 10(f). 
Furthermore, we use NC as a quality indicator to verify the robustness of the proposed zero2 watermarking algorithm for key-frames. According to Table 6 and Table 7, the NC values obtained from 3 watermark under the noise attacks and filtering attacks are all very high. Under noise attacks, the highest $4 \mathrm{NC}$ value is 0.9979 and the lowest is 0.9645 , with the average value is 0.9812 . Under filtering attacks, 5 the highest NC value is 0.9997 and the lowest NC value is 0.9903 .

6 Moreover, in order to reflect the changes of the algorithm in different intensities under noise attacks, 7 five kinds of salt and pepper noise and multiplicative noise attacks with different intensities are carried 8 out. The changes of NC values under different attacks intensities are shown in Fig. 11. According to Fig.

911 , for noise attacks, the greater the noise intensity is, the lower the NC value is.

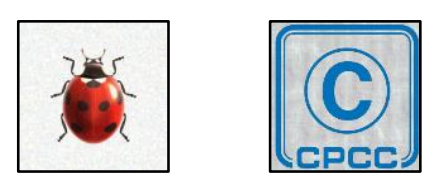

(a) Attack of salt and pepper noise (0.03) and the extracted watermark

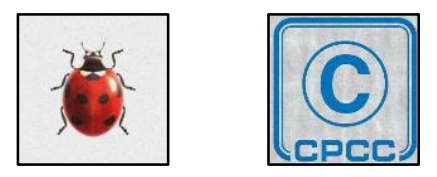

(b) Attack of gaussian noise (0.03) and the extracted watermark

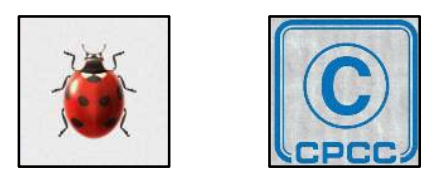

(c) Attack of multiplicative noise (0.03) and the extracted watermark

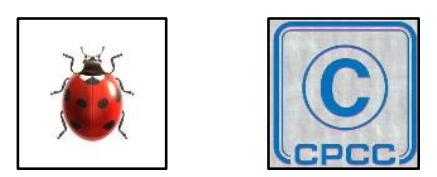

(d) Attack of sharpening and the extracted watermark

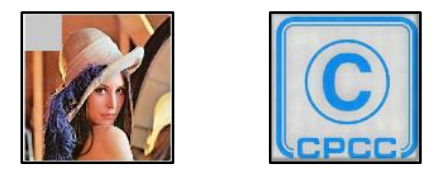

(e) Attack of 1/16 cropping in the upper left corner and the extracted watermark
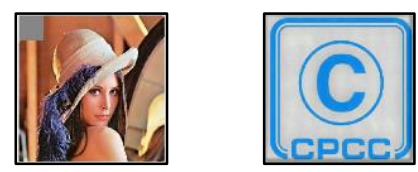

(f) Attack of $1 / 32$ cropping in the upper left corner and the extracted watermark

Fig. 10 Results of simulation attack 
Table 6 NC values obtained from watermark of the Fig. 10a-e under noise attacks

\begin{tabular}{ccccccc}
\hline Attack Types & Parameters & Lena & Vegetable & Animal & Bag & Ant \\
& & & & & & \\
Sault and pepper noise & 0.01 & 0.9958 & 0.9957 & 0.9946 & 0.9942 & 0.9963 \\
& 0.03 & 0.9870 & 0.9868 & 0.9864 & 0.9875 & 0.9885 \\
Gaussian noise & 0.05 & 0.9801 & 0.9811 & 0.9807 & 0.9809 & 0.9789 \\
& 0.01 & 0.9914 & 0.9907 & 0.9908 & 0.9926 & 0.9932 \\
Multiplicative noise & 0.03 & 0.9785 & 0.9767 & 0.9777 & 0.9789 & 0.9765 \\
& 0.05 & 0.9667 & 0.9639 & 0.9656 & 0.9645 & 0.9658 \\
& 0.01 & 0.9981 & 0.9973 & 0.9978 & 0.9975 & 0.9979 \\
& 0.03 & 0.9915 & 0.9910 & 0.9946 & 0.9934 & 0.9961 \\
& 0.05 & 0.9856 & 0.9838 & 0.9919 & 0.9894 & 0.9876 \\
\hline
\end{tabular}

Table 7 NC values obtained from watermark under filtering attacks

\begin{tabular}{ccccccc}
\hline Attack Types & Parameters & Lena & Vegetable & Animal & Bag & Ant \\
\hline \multirow{2}{*}{ Mean filtering } & $3 * 3$ & 0.9975 & 0.9989 & 0.9977 & 0.9993 & 0.9947 \\
& $5 * 5$ & 0.9944 & 0.9975 & 0.9965 & 0.9959 & 0.9976 \\
& $7 * 7$ & 0.9918 & 0.9960 & 0.9953 & 0.9929 & 0.9903 \\
Wiener filtering & $3 * 3$ & 0.9994 & 0.9998 & 0.9995 & 0.9997 & 0.9988 \\
& $5 * 5$ & 0.9987 & 0.9995 & 0.9988 & 0.9987 & 0.9993 \\
\multirow{2}{*}{ Median filtering } & $7 * 7$ & 0.9980 & 0.9993 & 0.9979 & 0.9978 & 0.9996 \\
& $3 * 3$ & 0.9986 & 0.9996 & 0.999 & 0.9978 & 0.9993 \\
& $5 * 5$ & 0.9966 & 0.9991 & 0.9979 & 0.9964 & 0.9983 \\
& $7 * 7$ & 0.9950 & 0.9985 & 0.9965 & 0.9959 & 0.9975 \\
\hline
\end{tabular}

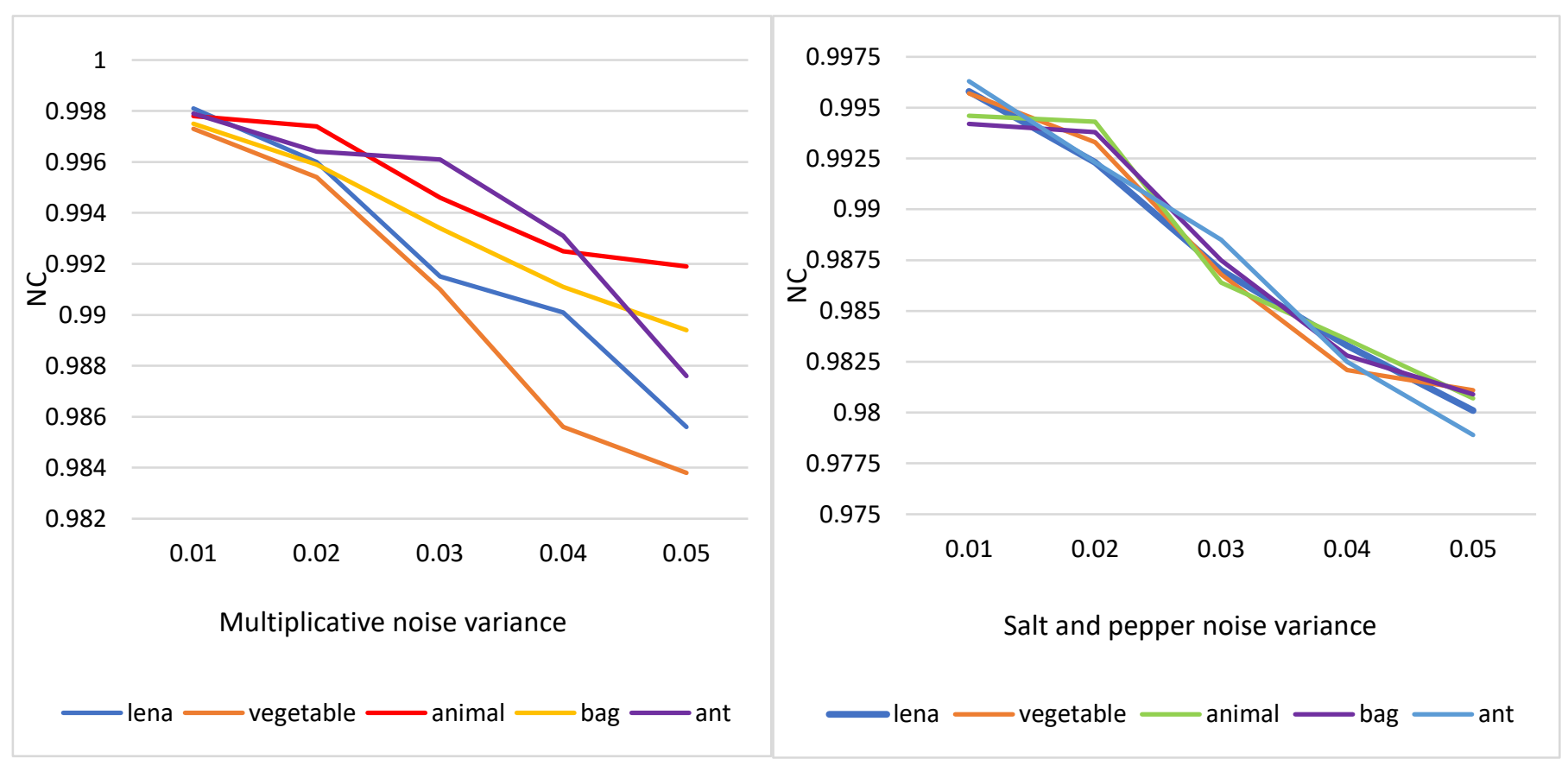

(a)

(b)

Fig. 11 Attack of multiplicative noise and salt and pepper noise variance 


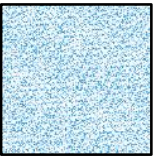

(a)

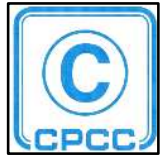

(b)

Fig. 12 zero-watermark and correctly extracted watermark

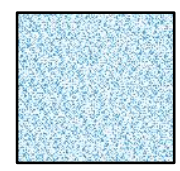

(a)

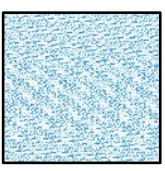

(b)

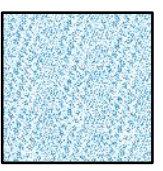

(c)

Fig. 13 watermark extracted by different parameters of Arnold Transform

1

\subsubsection{Security}

In this subsection, we sets three comparative experiments to verify the security by Arnold transform. In Fig. 12, the zero-watermark is obtained by adding Fig. 8(e) to Fig. 8(f), and watermark is correctly extracted by right secret Keys. Fig. 13(a) is extracted by wrong Keys. Fig. 13(b) is extracted by wrong scrambling rounds. Fig. 13(c) is extracted by wrong Keys and scrambling rounds.

\subsection{Blockchain system}

In order to verify the availability of applying blockchain to storage of zero-watermarks, this part tests the response time of the blockchain system to represent its actual efficiency, and illustrate the feasibility and rationality of the combination of the system and the blockchain.

Response time of blockchain system is one of the important indicators of system performance. The main functions of the blockchain system in proposed scheme are on-chain data entry and on-chain data traceability. Therefore, the data volume of on-chain traceability information is the key to affecting response time of the system. Fig. 14 shows the relationship between data volume of on-chain traceability information and the system response time. When the data on the chain increases from $1 \mathrm{G}$ to $8 \mathrm{G}$, the system response time increases from $231 \mathrm{~ms}$ to $511 \mathrm{~ms}$.

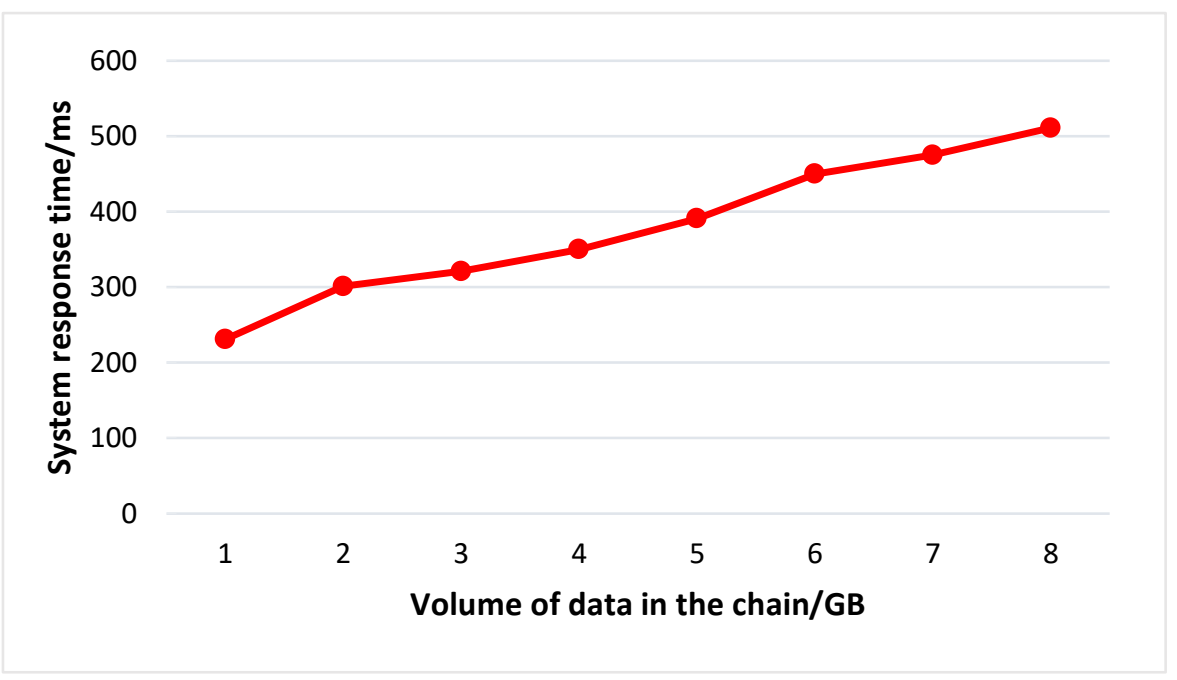

Fig. 14 Response time 


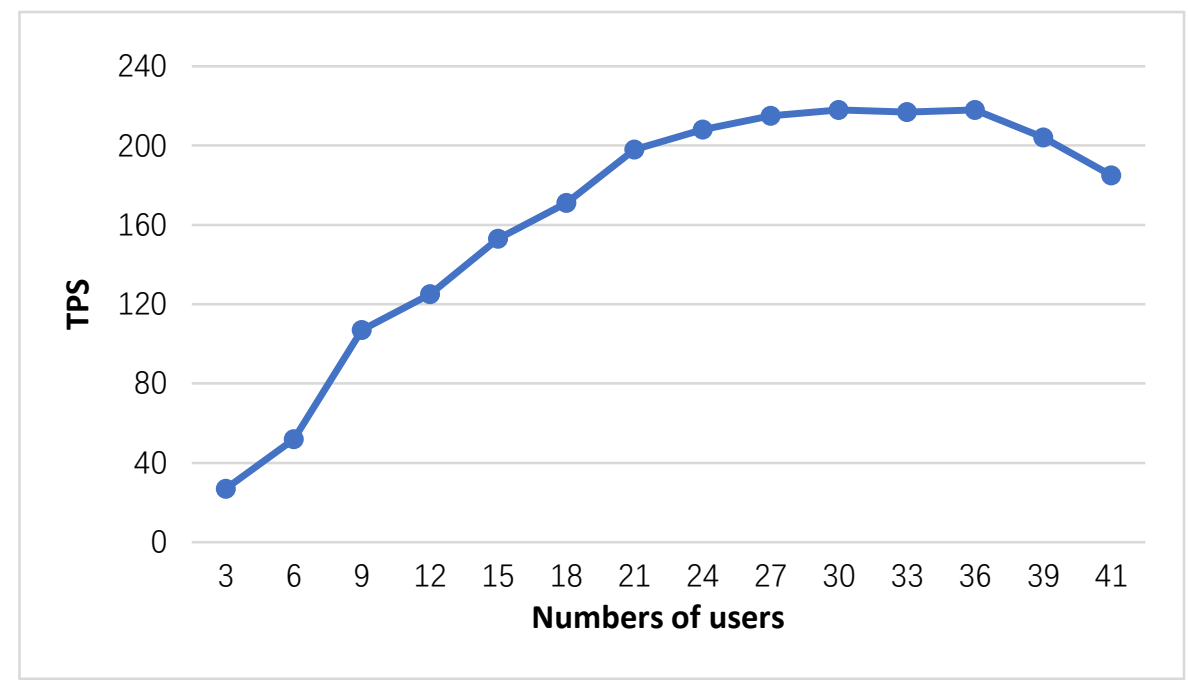

Fig. 15 Throughput

Our scheme uses multi-threaded programming for performance testing, using each thread to simulate a user (client) interacting with the blockchain system, and conducting multiple sets of tests. Each time of test adds 3 users, and experimental data such as response time and number of requests are recorded to calculate the throughput of the system under different loads. Meanwhile, we have to monitor the server memory, CPU, network, disk read/write and other resource usage. The test is not stopping until TPS (Transaction Per Second, number of concurrent/average response time) reaches its peak. The experimental results are shown in Fig. 15. The system has not yet reached its peak when the number of simulated requests is between 3 and 27. Simultaneously, the TPS boost is stable. TPS stabilizes after the number of users exceeds 27 , but decreases when it reaches 36 .

\subsection{Analysis}

From the experimental results in Table 2, it can be seen that key-frames extraction algorithm works well for various videos. Although a small number of missed detection and false detection exist.

From the above experimental results on zero-watermarking algorithm, the proposed zerowatermarking algorithm in this paper can effectively avoid false alarm. Furthermore, our zero-watermark algorithm combined with NSCT-SVD is more robust than the traditional DCT-SVD method. Due to the combination of NSCT and SVD, the change in the low frequency coefficients of each sub-block is small under noise attacks and filtering attacks. The results show that the algorithm is robust under noise attacks and filtering attacks. Due to the block processing when constructing the feature matrix and SVD of each sub-block, so that proposed zero-watermarking algorithm can effectively resist local cropping attack. From the above experimental results, it can be seen that as long as the Keys or scrambling rounds is incorrectly, the correct watermark cannot be obtained, which fully verifies the security of Arnold Transform in proposed zero-watermarking algorithm.

According to the experimental results on blockchain system, the delay of the blockchain system is within range of technical standards of the blockchain industry. Assuming the zero- watermark is $50 \mathrm{kB}$, the data volume of the $18 \mathrm{~min}$ short video on the chain is $71 \mathrm{MB}$. When the amount of data on the chain increases to $80 \mathrm{~GB}$, the system can register $18 \mathrm{~min}$ videos about 1153 , while the response time of the blockchain system is still a few seconds. In the process of testing TPS, the utilization rate of each hardware was below $100 \%$. After comparison and inspection, it may be caused by the performance 
limitation of Fisco Bcos. The experimental results show that the TPS of the blockchain system is about 216, which not only meets the performance indicators of the blockchain network, but also meets the needs of the video copyright protection scheme.

\section{Discussion}

Before discussing the proposed video zero-watermarking algorithm, we compare the traditional blind watermarking algorithm with zero-watermarking algorithm. The traditional blind watermarking scheme is embedding the features of watermark into key-frames. The zero-watermarking algorithm is embedding the features of key-frames into the watermark to generate zero-watermark. Copyright protection is realized by registering zero-watermark with features of key-frames. From the perspective of visual quality, the traditional blind watermarking is embedding the watermark into the key-frames, so keyframes in the video will inevitably produce noise. However, zero-watermarking will not take actions on key-frames so that it will not influence video quality. From the perspective of security, the traditional blind watermark is easy to be detected and destroyed, even erased. But the zero-watermarking is encrypted and stored in a trusted third-party, makes it hard for attackers to know where to begin.

In the proposed zero-watermarking algorithm, a colour video will generate many zero-watermarks from extracted key-frames. The zero-watermarks generated by multiple key-frames can effectively resist malicious clipping of key-frames in the actual state of secondary creation, and prevent key-frames being clipped to extract effective feature matrices, so as to get the copyright watermarks. By this way, even if the key-frames are clipped, watermarks which are extracted still keep a good clarity. Besides, there are twice chunking in the generation of feature matrices which belongs the proposed zero-watermarking algorithm. The first processing is before NSCT. When the colour video is attacked by cropping attack, the coefficients after NSCT can be independent of each other. The second processing makes the subsequent SVD more stable. In addition, the decomposition of RGB in this algorithm enhances security of the system, because the three zero-watermarks are required to extract a colour watermark, and the decomposition of RGB achieves a triple encryption logically with Arnold Transform. Above all, the fundamental feature of the zero-watermarking algorithm is that copyright protection is realized without any modification of the video. This zero-watermarking algorithm generates Key $K_{1}$, which will be kept by the owner of video copyright. Key $K_{2}$ can be uploaded to the blockchain network. In process of copyright certification, the user provides the Key $K_{1}$ to match the key pairs $\left(K_{1}, K_{2}\right)$ so as to extract copyright watermarks from zero-watermarks on the blockchain.

In the video copyright protection system, the blockchain network replaces the third-party notary of the traditional zero-watermarking algorithm and avoids the security issues existed in third-party notaries. Furthermore, excellent security control mechanisms exist in the blockchain. The system applied in the field of video copyright protection have high demand for data security, so access control of data on the alliance chain is required, whose strategy is mainly divided into the following two aspects.

- Access control of communication data on the chain is accomplished through node certificates and SSL.

- In the access control of node storage data, the blockchain uses disk encryption, which is performed within the institution. In the institution's intranet, each institution independently encrypts the node's rigid disk data. When the rigid disk of the machine is away from the institution 
and the node is allowed to start up on a network outside the institution's intranet. The rigid disk data will not be decrypted and the node will not be able to start up and thus steal the data on the federated chain.

In addition, the data on the chain (the colour zero-watermark) has been scrambled. Even if being accessed, no valuable information can be obtained. Copyright rights transactions will only be done on the blockchain network without any further manipulation on the video. This way is more efficient than traditional methods. For example, all permission transactions and permission ownership information will be recorded on the blockchain network and be protected from malicious tampering. Thus, the malicious user uses and reproduces a video illegally, a legally valid proof will be found through fast traceability in the video copyright protection system.

\section{Conclusions}

Aiming at the problems of the traditional watermarking scheme for video copyright, such as poor robustness, weak imperceptibility and single function, we propose a zero-watermarking protection scheme for video copyright based on blockchain and NSCT + SVD.

In our scheme, firstly, the proposed key-frames extraction algorithm performs high accuracy in filtering key-frames. Secondly, we choose the NSCT which is combined with the Nonsubsampled Pyramid (NSP) and the Nonsubsampled Directional Filter Bank (NSDFB) in zero-watermarking algorithm. This method can decompose the images in multi-scale and multi-direction, making up for the deficiency that wavelet transform only has three directions of vertical, horizontal and diagonal transformation of the image. It has good translation invariance, making up for the shortcomings of contourlet transform sampling. Thirdly, zero-watermarking algorithm based on SVD after chunking can achieve the stability in the process of zero-watermarking, and zero-watermarking method makes video quality not be affected. Fourthly, the difficulties in security and efficiency of video copyright protection are overcome by combining blockchain and the video zero-watermarking algorithm.

Further works are as follows.

- Investigate a low-cost copyright protection method based on blockchain and digital watermarking technology for derivative work problem (The malicious person modifies the video and then claims to be original).

- Investigate a combination system of IPFS and blockchain to increase the storage capacity on the blockchain.

- Study cross-chain key technologies so as to realize protection of cross-domain video copyright.

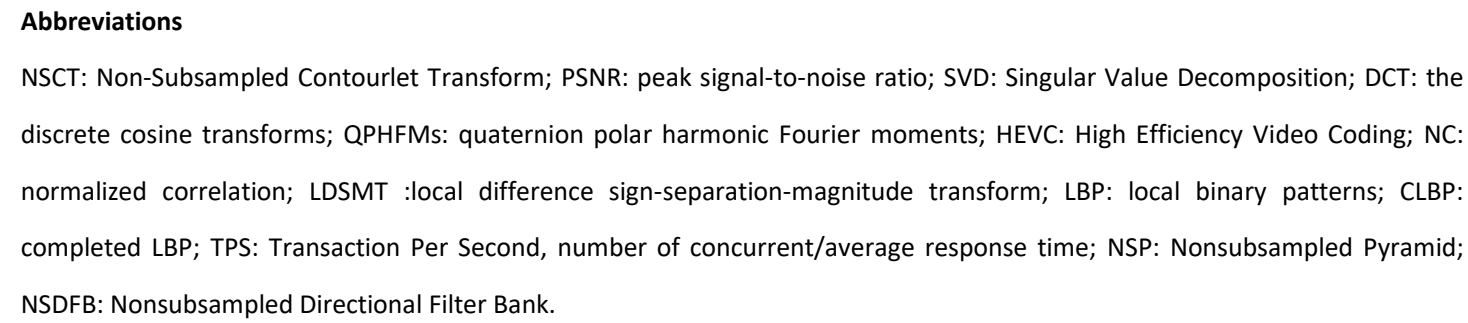




\section{Authors' contributions}

Xiangqi Wu and Peng Ma conceived and designed this research together. Xiangqi Wu designed the framework of this program. Peng Ma designed the core algorithm. Zihan Jin and Yuxuan Wu investigated and wrote the paper. Wei Ou Provide financial sup port and guidance. All authors reviewed and approved final manuscript.

\section{Funding}

Not applicable.

\section{Availability of data and materials}

Not applicable

\section{Declarations}

\section{Competing interests}

The authors declare that they have no competing interests.

\section{Author details}

${ }^{1}$ School of Cyber Security (School of Cryptology), Hainan University, 58 Renmin Avenue, Meilan District, Haikou 570228, China.

\section{References}

1. H. Gao, C. Liu, Y. Li and X. Yang, V2VR: Reliable hybrid-network-oriented V2V data transmission and routing considering RSUs and connectivity probability. IEEE Transactions on Intelligent Transportation Systems (2020). doi: 10.1109/TITS.2020.2983835.

2. Xia W, Research status and development of digital watermarking. The Guide of Science \& Education. 3,161-161(2017).

3. Zhao Q, Digital watermarking copyright protection system. (Capital University of Economics and Trade, Beijing, 2004).

4. Atsushi, KOBAYASHI, Akira, HASEGAWA, Miho, FUKUDA, A practice of copyright protection applying digital watermark technology: studying applications in still pictures and moving pictures, and exploration of future expectations. Journal of Information Processing \& Management (2017). doi:10.1241/johokanri.60.89.

5. He F, Digital watermarking and its anti-compression robustness test based on wavelet domain. Automation and Information Engineering. 44(5), 42-44+50 (2020).

6. Zai R, WANG, Babak, SHIRI, Dumitru, BALEANU, Discrete fractional watermark technique. Frontiers of Information Technology \& Electronic Engineering. 6, 880-883 (2020).

7. Li Y, Wei D, Zhang L, Double-encrypted watermarking algorithm based on cosine transform and fractional Fourier transform in invariant wavelet domain - science direct. Information Sciences (2020).

8. Wen Q, Sun T, Wang S, Concept and application of zero-watermarking. Journal of Electronics. 2,214-216(2003).

9. Wang $\mathrm{C}$ et al, Stereo image zero-watermarking algorithm based on ternary polar harmonic Fourier moments and chaotic mapping. Information Sciences. 450, 141-156 (2018).

10. Xue H, Chi W, Liu X, A zero-watermarking algorithm based on NSST and Hessenberg decomposition. Modern Computer. $10,89-93+103(2020)$.

11. Amiri, Ali, and S. Mirzakuchaki, A digital watermarking method based on NSCT transform and hybrid evolutionary algorithms with neural networks. SN Applied Sciences. 2(10), 1-15(2020).

12. Xuan M, H. 264/AVC video integrity authentication key technology research. (Hefei University of Technology, Hefei, 2011). 
13. Meng H, Research on video encryption technology based on H. 264 coding standard. (Northeast University of China, Liaoning, 2020).

14. Liu X, An overview of video digital watermarking technology. Television Technology. 44(5), 11-15 (2020).

15. Sun $Y$ et al, Research on scalable video watermarking algorithm based on H. 264 compressed domain. Optik (2020).

16. Qiu Y, Xue F, Tang J, Spatio-temporal feature analysis combined with random key for compressed domain digital video watermark embedding and extraction algorithm. Computer Applications Researchvol. 36(9), 2813-2817 (2019).

17. Guo X, Block chain technology rebuilds the new order of media industry -- take baidu totem as an example. Science and Technology Communication. 12(9), 4-6+24 (2020).

18. Ruan X, Research on financial problems of supply chain finance model embedded based on block chain technology -- a case study of ant financial double-chain communication. Shanxi Agricultural Economics. 10, 162-163 (2020).

19. Guo Z, Zhang L, Zhang D, A completed modeling of local binary pattern operator for texture classification. IEEE Transactions on Image Processing. 19(6), 1657-1663 (2010).

20. Ojala, T, M. Pietikainen, T. Maenpaa, Multiresolution gray-scale and rotation invariant texture classification with local binary patterns. IEEE Transactions on Pattern Analysis \& Machine Intelligence. 24(7), 971-987 (2002).

21. Q Kou et al, Texture target classification integrating CLBP and local geometric features. Photoelectric engineering. 46(11), 66-73 (2019).

22. $\mathrm{Xu} \mathrm{S}$ et al, Texture descriptors for spatial statistical features based on LBP values. Pattern Recognition and Artificial Intelligence 26(8), 769-776 (2013).

23. Yang J et al, Research on undersampling methods based on data density distribution. Computer Applications Research. 33(10), 2997-3000 (2016).

24. Liu J, Zhao W, Zhu H, Pattern recognition: pattern recognition. Harbin Institute of Technology Press (2014).

25. Deng $S$, Robust watermarking algorithm for wavelet transform and singular value decomposition. Laser Journal. 36(4), 8689 (2015).

26. Guo L et al, Fingerprint watermarking authentication algorithm based on SM2 and singular value decomposition (SVD). Journal of Beijing Institute of Printing. 27(2), 46-50 (2019).

27. Hao X, Peng G, Video summarization based on SVD and sparse subspace clustering. Journal of Computer-Aided Design and Graphics. 29(3), 485-492 (2017).

28. Xiao $Z$ et al, Enhanced singular value decomposition and cellular neural networks for zero-watermarking. Chinese Journal of Graphical Graphics. 3, 1144 - 1148+1153 (2017).

29. Wang $L$ et al, Digital watermarking algorithm in wavelet domain based on Arnold scrambling and logistic chaotic encryption. Information Technology. 2018, 49 - 53+58 (2018).

30. Chen $Y$, Research on colour image encryption algorithm based on chaotic random chunking and Arnold transform. Digital Technology and Applications. 37(6), 135-137 (2019).

31. Wu L, Zhang J et al, Arnold transform and its inverse transform. Microcomputer Information. 26(14), 206-208 (2010).

32. Guo L et al, Fingerprint watermarking authentication algorithm based on SM2 and singular value decomposition (SVD). Journal of Beijing Institute of Printing. 27(2), 46-50 (2019).

\section{Figure title and legend}

Fig. 1 Architecture of the protection system. It shows the whole process about USER A's registration and verification. And it includes the USER B' get-permission and verification;

Fig. 2 Description of CLBP algorithm I. The matrix of sampling block and its local difference are (a) and (b);

Fig. 3 Description of CLBP algorithm II. Sign components, and gradient components are (a) and (b); 
Fig. 4 CLBP algorithm. Original image is classed by CLBP;

Fig. 5 Process of clustering algorithm. It demonstrates from the input of the video to the determination of the final k, clustering centre set and clustering;

Fig. 6 Process of watermark generation. The watermark information is encrypted and embedded in the key frame;

Fig. 7 Process of watermark extraction. The watermark information is decrypted and extracted from the key frame;

Fig. 8 Images and copyright watermark. All images used in our scheme;

Fig. 9 Zero-watermark after extraction. (a) and (b) generate a zero-watermark image (c);

Fig.10 Results of simulation attack. The result of various attacks on the image;

Fig. 11 Attack of multiplicative noise and salt and pepper noise variance. Five kinds of multiplicative noise attacks (a) and salt and pepper noise attacks (b) with different intensities are carried out. Blue, orange, red, yellow and purple represent lena, vegetable, animal, bag, and ant images;

Fig. 12 Zero-watermark and correctly extracted watermark. The result of successfully extracting the watermark;

Fig. 13 watermark extracted by different parameters of Arnold Transform. Watermark results extracted by different keys incorrectly

Fig. 14 Response time. Changes in response time as the volume of data increases;

Fig. 15 Throughput. Changes in TPS as the number of users increases; 